\title{
STOCHASTIC MODELLING OF INSULIN SENSITIVITY VARIABILITY IN CRITICAL CARE
}

\author{
J. Lin ${ }^{1}$, D. Lee ${ }^{2}$, J. G. Chase ${ }^{1}$, G. M. Shaw ${ }^{3}$, C. E. Hann ${ }^{1}$, T. Lotz ${ }^{1}$, X.-W. Wong ${ }^{1}$ \\ ${ }^{1}$ Dept of Mechanical Engineering, University of Canterbury, Christchurch, New Zealand \\ ${ }^{2}$ Dept of Mathematics and Statistics, University of Canterbury, Christchurch, New Zealand \\ ${ }^{3}$ Dept of Intensive Care Medicine, Christchurch Hospital, Christchurch, New Zealand
}

geoff.chase@canterbury.ac.nz

\begin{abstract}
Hyperglycaemia is prevalent in critical care, and tight control can reduce mortality by 29 45\%. Targeted glucose control can be achieved by frequent fitting and prediction of a modelled insulin sensitivity index, $S_{I}$. This parameter varies significantly in the critically ill due to condition evolution and drug therapies. A 3-D stochastic model of hourly $S_{I}$ variability is constructed using retrospective data from 18 long term critical care patients. The model can be used to produce the blood glucose level probability distribution one hour following a known insulin and/or nutrition intervention. Thus, it enables accurate prediction for glycemic control based on confidence intervals.
\end{abstract}

\section{Introduction}

Hyperglycemia and severe insulin resistance are prevalent in the critically ill, even given no history of diabetes [1-3]. Tight glucose control has been shown to reduce Intensive Care Unit (ICU) patient mortality by up to $45 \%$ [1-3]. Therefore, tight regulation glycemic control algorithms in the ICU would reduce mortality and the burden on time and medical resources.

Previous clinical glycemic control studies [4] focused on critical care patients, whose glucose-insulin dynamics are highly variable due to the stress from their condition and the impact of drug therapies. Chase et al. [4] developed a control algorithm that has been clinically verified in the ICU to predicatively reduce hyperglycaemic levels, while accounting for interpatient variability and evolving physiological condition. The overall approach is a targeted adaptive control scheme that identifies patient dynamics, particularly with respect to insulin sensitivity.

Following Chase et al. [4], better understanding and modelling of patient variability in the ICU can lead to better glycemic management. In particular, a common risk in intensive insulin therapies in the ICU, is hypoglycemic events $[1,5]$. Understanding and modelling the variability in patient condition, or more specifically, the patients' variable dynamic response to insulin, will thus assist clinical control intervention decision making, and minimise the associated risk.

Therefore, the ultimate goal of this study is to produce blood glucose confidence bands for control intervention decisions based on stochastic models of clinically observed parameter variations. These bands allow targeted control with user specified confidence on the outcome. The result will be added certainty and safety in glycemic control.

\section{Glucose-Insulin System Model}

This study uses a patient-specific, two-compartment glucose-insulin system model from Chase et al. [4] and Hann et al. [6]. This physiologically verified model, which captures the fundamental dynamic responses to elevated glycemic levels and insulin, accounts for timevarying insulin sensitivity and endogenous glucose removal, along with two different saturation kinetics.

$$
\begin{aligned}
& \dot{G}=-p_{G} G-S_{I}\left(G+G_{E}\right) \frac{Q}{1+\alpha_{G} Q}+P(t) \\
& \dot{Q}=-k Q+k I \\
& \dot{I}=-n \frac{I}{1+\alpha_{I} I}+\frac{u_{e x}(t)}{V_{I}}
\end{aligned}
$$

where $G$ and $I$ denote the glucose above an equilibrium level, $G_{E}$, and the plasma insulin from an exogenous insulin input respectively. Insulin utilization over time is $Q$, where $k$ is the effective insulin decay rate. Patient endogenous glucose removal and insulin sensitivity are $p_{G}$ and $S_{I}$. Insulin distribution volume is $V_{I}$ and $n$ represents plasma insulin decay. External nutrition and insulin input are $P(t)$ and $u_{e x}(t)$. Michaelis-Menten saturation in plasma insulin disappearance and insulinstimulated glucose removal are defined by $\alpha_{I}$ and $\alpha_{G}$.

Insulin sensitivity, $S_{I}$, is the critical parameter that drives the dynamics of the system. It represents the tissue insulin sensitivity, and its variation shows the evolution of patient condition and drug therapy. Identifying $S_{I}$ and its changes over time is thus critical to providing safe and effective tight glycemic control in the highly variable critical care population.

\section{Integral-Based Parameter Identification}

Using generic values for $\alpha_{G}, \alpha_{I}, n, k$ and $V_{I}$ limits the model unknowns to $p_{G}$ and $S_{I}$, as shown in Equations (1)-(3). An integration-based parameter identification method presented in Hann et al. [6] is used for accurate identification of patient specific parameters, $p_{G}$ and $S_{I}$. 
The functions for $p_{G}$ and $S_{I}$ can be arbitrarily defined. The integral-based method presents a convex least squares problem that demands little computational effort. In contrast, the commonly used non-linear recursive least squares routine is non-convex and starting point dependent [7]. Details of the fitting routine are outlined in Hann et al. [6].

\section{Stochastic Modelling}

Patient parameters, $p_{G}$ and $S_{I}$ are fitted to long term retrospective clinical data from 18 patients in the Department of Intensive Care Medicine, Christchurch Hospital. These patients are a random selection from a 201-patients data audit [8]. Each patient record spans at least one day with data every three-hours or less. This cohort broadly represents the cross section of patients seen in the ICU, regarding medical condition, age, sex, APACHE II scores and mortality. Diabetic patients are somewhat over-represented as they receive greater attention in blood glucose levels.

Zero order piecewise linear functions are used to define $p_{G}$ and $S_{I}$, with a discontinuity every two hours for $p_{G}$ and every hour for $S_{I}$. Greater variability is given to $S_{I}$ because studies have shown that $S_{I}$ is much more variable than $p_{G}$ [6]. Table 1 shows the values used for the generic parameters based on various studies [9-11].

Table 1. Generic parameter values [9-11]

\begin{tabular}{c|c|l} 
Parameter & Unit & Value \\
\hline \hline$\alpha_{G}$ & $L / m U$ & $1 / 65$ \\
\hline$\alpha_{I}$ & $L / m U$ & 0.0017 \\
\hline$n$ & $\mathrm{~min}^{-1}$ & 0.16 \\
\hline$k$ & $\mathrm{~min}^{-1}$ & 0.0198 \\
\hline$V_{I}$ & $L$ & 3.15
\end{tabular}

The fitted long term $p_{G}$ and $S_{I}$ data reveals that the variability of both parameters is dependent on its present value. The distribution of fitted $S_{I}$ is shown by the dots in Figure 1. The probability distribution of potential $S_{I}$, shown by the probability bands, clearly varies with its value across the horizontal axis.

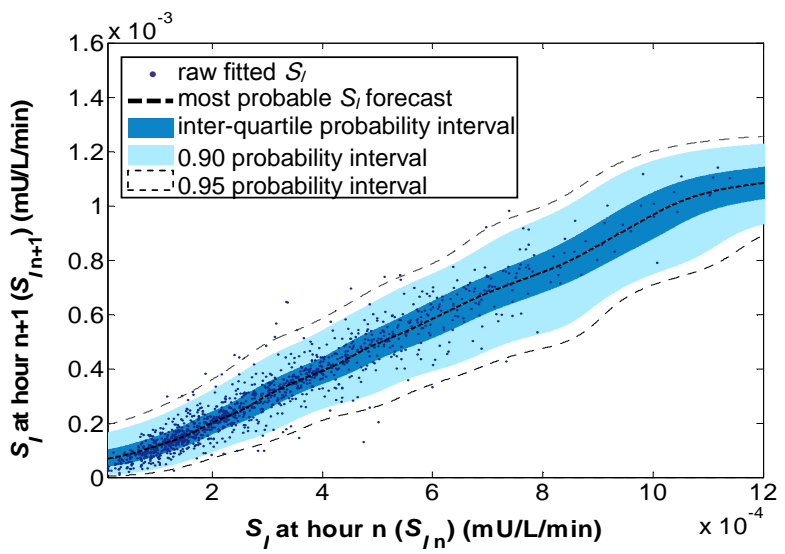

Figure 1. Fitted $S_{I}$ and probability intervals

Thus, the variations in $S_{I}$ can be treated as a Markov process. A Markov process has the property that the conditional probability distribution of future states of the process, given the present state, depends only upon the current state. Therefore, using the Markov property of the stochastic behaviour of $S_{I}$, the conditional probability distribution of $S_{I n+1}$ taking on a value $y$ can be calculated by knowing $S_{I n}=x$ :

$$
P\left(S_{I n+1}=y \mid S_{I n}=x\right)=\frac{P\left(S_{I n}=x, S_{I n+1}=y\right)}{P\left(S_{I n}=x\right)}
$$

Considering the fitted $S_{I}$ in a 2-D space, as shown in Figure 1, the joint probability function across the $x-y\left(S_{I}\right.$ $\left.{ }_{n}-S_{I n+1}\right)$ plane is defined by the fitted values shown by the dots whose coordinates are $x_{i}$ and $y_{\mathrm{i}}$,

$$
\begin{aligned}
& P(x, y)=\frac{1}{n} \sum_{i=1}^{n} \frac{\phi\left(x ; x_{i}, \sigma_{x_{i}}{ }^{2}\right)}{p_{x_{i}}} \frac{\phi\left(y ; y_{i}, \sigma_{y_{i}}{ }^{2}\right)}{p_{y_{i}}} \\
& p_{x_{i}}=\int_{0}^{\infty} \phi\left(x ; x_{i}, \sigma_{x_{i}}{ }^{2}\right) \\
& p_{y_{i}}=\int_{0}^{\infty} \phi\left(y ; y_{i},{\sigma_{y_{i}}}^{2}\right)
\end{aligned}
$$

Effectively, the 2-D joint probability function is the normalised summation of normal probability distribution functions $\phi\left(x ; x_{i}, \sigma_{x_{i}}{ }^{2}\right)$ centred at individual data points.

To illustrate a 3-D map in the mind, consider this numerical operation as a sand building exercise. If the first quadrant of the $x-y\left(S_{I n}-S_{I n+1}\right)$ plane, as shown in Figure 1, is where the sand box is confined in, and that a pile of sand of a cubic unit is dropped onto every dot in Figure 1, then the resulted sand sculpture is the simple representation of the joint probability $P(x, y)$ on the $x-y$ $\left(S_{I n}-S_{I n+1}\right)$ plane. In Equations (5)-(7), the variance $\sigma$ at each data point is a function of the local data density in a centred and orthonormalised space of $x$ and $y$. Putting Equations (6) and (7) into Equation (5) normalises each $\phi\left(x ; x_{i}, \sigma_{x_{i}}{ }^{2}\right)$ and $\phi\left(y ; y_{i}, \sigma_{y_{i}}{ }^{2}\right)$ in the positive domain. This, in the sand building exercise example, effectively puts boundaries along $x=0$ and $y=$ 0 , confining sand to stay in the first quadrant, and therefore forces physiological validity in $S_{I}$ values.

In Equation (4), the denominator in the right hand side can be calculated by integrating Equation (5) with respect to $y$. Hence, Equation (5) can be calculated:

$$
\begin{aligned}
& P\left(S_{I n+1}=y \mid S_{I n}=x\right)=\sum_{i=1}^{n} \omega_{i}(x) \frac{\phi\left(y ; y_{i}, \sigma_{y_{i}}{ }^{2}\right)}{p_{y_{i}}} \\
& \omega_{i}(x)=\frac{\phi\left(x ; x_{i}, \sigma_{x_{i}}{ }^{2}\right) / p_{x_{i}}}{\sum_{j=1}^{n} \phi\left(x ; x_{j}, \sigma_{x_{j}}{ }^{2}\right) / p_{x_{i}}}
\end{aligned}
$$

Thus, knowing $S_{I n}=x$ at hour $n$, the probability of $S_{I n+1}=y$ at hour $n+1$ can be calculated using Equations (8) and (9). The probability intervals shown in Figure 1 are also calculated from integrating Equation (8). 
Plotting Equation (8) across the $x-y\left(S_{I n}-S_{I n+1}\right)$ plane, the 3-D stochastic model is shown in Figure 2.

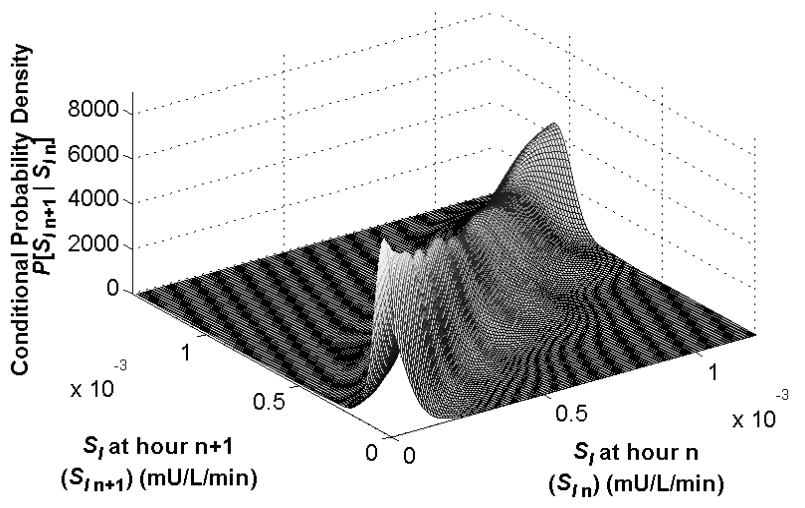

Figure 2. Three-dimensional $S_{I}$ stochastic model

The same numerical operations described in Equations (4)-(9) also apply to $p_{G}$, producing similar results. However the probability density across the $x-y$ plane is highly concentrated along the line $y=x$. This result reinforces the idea that $p_{G}$ is effectively constant, even though patient specific [6]. Hence, variability of $p_{G}$ is neglected in this study.

The stochastic parameter model can be integrated into the glucose-insulin system model of Equations (1)(3). This step allows the blood glucose level probability distribution one hour following a known insulin and/or nutrition intervention to be defined. The stochastic model therefore enables more knowledgeable predictions with defined probability distributions for the outcomes of glycemic control inputs.

\section{Validation on Clinical Control Trials}

The stochastic model developed from the 18-patients cohort in Hann et al. [6] is evaluated on 8 previous clinical control trials in the ICU [12] that are independent from the cohort used to develop the stochastic model. The trials performed consist of hourly cycles of the following steps [12]:

1. Measure blood glucose levels.

2. Fit $p_{G}$ and $S_{I}$ to the measured blood glucose.

3. Determine new control intervention to achieve a blood glucose target level.

4. Implement control intervention.

To asses the stochastic model, these 8 trials are numerically re-enacted with control interventions as given in the trial and step 3 of the trial cycle modified to:

3a. Generate potential $S_{I}$ probability intervals from the time-average identified $S_{I}$ of the fitted time interval using the stochastic model.

3b. Calculate blood glucose confidence intervals with respect to the $S_{I}$ probability intervals using the numerical model in Equations (1)-(3).
This test allows validation of the model in a clinical control scenario.

\section{Clinical Trial Review Results ad Discussion}

Blood glucose probability intervals were produced at each control intervention in the 8 clinical trials and compared to measured blood glucose levels one hour later. The results are shown in Table 2. Detailed result from Trial 4 is shown Figure 4.

Table 2. Retrospective probabilistic assessment on clinical control trials

\begin{tabular}{c|c|cc|cc}
$\begin{array}{c}\text { Clinical } \\
\text { control } \\
\text { patients }\end{array}$ & $\begin{array}{c}\text { Number of } \\
\text { interventions } \\
\text { made }\end{array}$ & $\begin{array}{c}\text { Measurement } \\
\text { error within } \\
\text { inter-quartile } \\
\text { probability } \\
\text { interval }\end{array}$ & $\begin{array}{c}\text { Measurement } \\
\text { error within } \\
0.90 \\
\text { probability } \\
\text { interval }\end{array}$ \\
\hline \hline 1 & 9 & 6 & $(67 \%)$ & 9 & $(100 \%)$ \\
2 & 9 & 5 & $(56 \%)$ & 7 & $(78 \%)$ \\
3 & 9 & 5 & $(56 \%)$ & 8 & $(89 \%)$ \\
4 & 9 & 5 & $(56 \%)$ & 7 & $(78 \%)$ \\
5 & 9 & 9 & $(100 \%)$ & 9 & $(100 \%)$ \\
6 & 9 & 9 & $(100 \%)$ & 9 & $(100 \%)$ \\
7 & 9 & 8 & $(89 \%)$ & 9 & $(100 \%)$ \\
8 & 23 & 16 & $(70 \%)$ & 23 & $(100 \%)$ \\
\hline total & 86 & 63 & $(73 \%)$ & 81 & $(94 \%)$
\end{tabular}

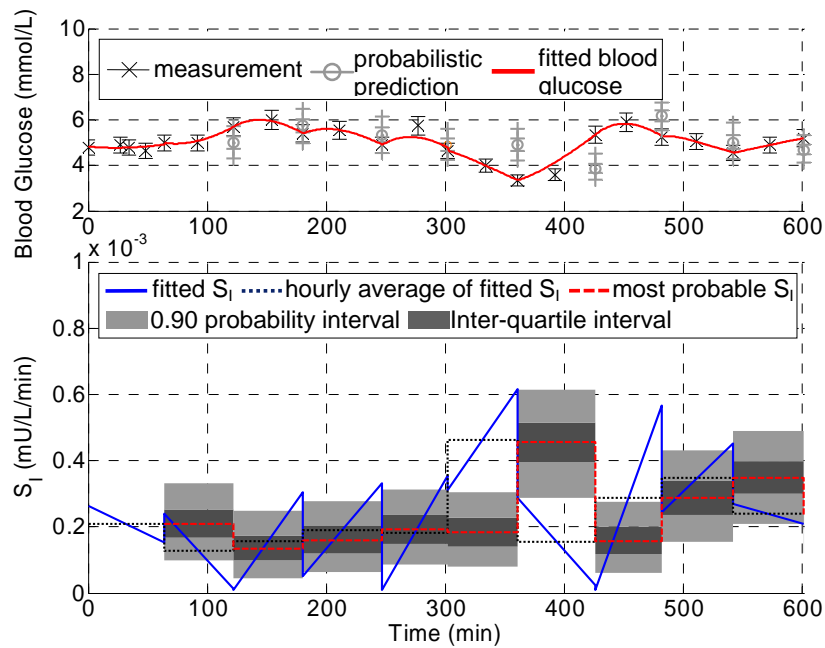

Figure 4. Simulated clinical control trial on Patient 4

The top panel of Figure 4 displays blood glucose, where the crosses are the actual clinical measurements with $7 \%$ measurement error, the solid line is the fitted blood glucose profile, and the circles are the most likely probabilistic blood glucose predictions following control interventions. The 0.90 and inter-quartile probability ranges are shown by the bars. The bottom panel shows the fitted $S_{I}$ and its probabilistic bounds produced from the stochastic model.

Patient 4's blood glucose briefly dropped below 4 $\mathrm{mmol} / \mathrm{L}$ during the trial, which was not accounted for in the probabilistic forecast. Having the identified Patient 4 $S_{I}$ profile, different control interventions were explored using the $S_{I}$ stochastic model to assist decision making. A comparison between the clinical trial and simulated new control intervention results is shown in Figure 5. 


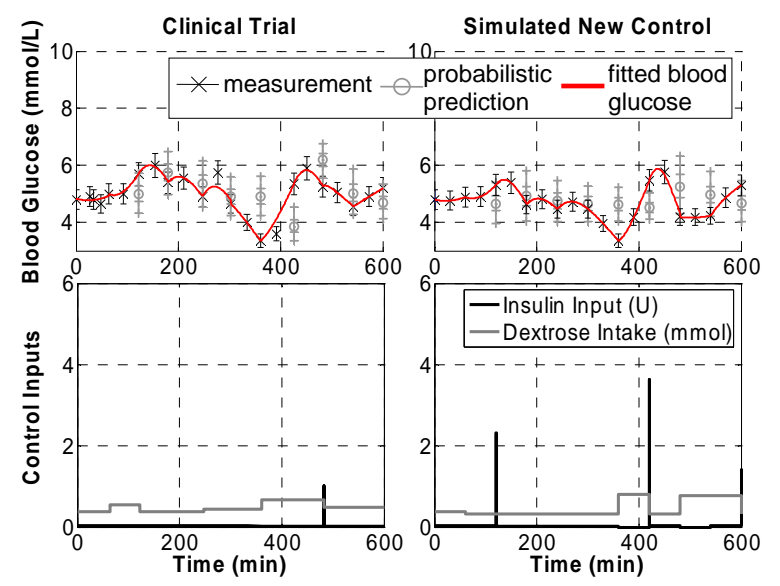

Figure 5. Clinical trial vs. simulated new control results on Patient 4

The simulated control aimed to maintain the glycemic levels close to but above $4 \mathrm{mmol} / \mathrm{L}$. More aggressive interventions were issued in the first half of the simulated trial, achieving more tightly controlled glycemic levels. The brief blood glucose excursion below $4 \mathrm{mmol} / \mathrm{L}$ was still unavoidable because the $S_{I}$ variation exceeded the 0.90 confidence limit of the stochastic model. A more vigorous remedy action was taken at 360 minutes in the simulated trial to obtain a 0.90 confidence in blood glucose above $4 \mathrm{mmol} / \mathrm{L}$ in an hour. Overall, the $S_{I}$ stochastic model can deliver tighter and safer glycemic management and improve control intervention selection.

Across the 8 patients, the $S_{I}$ stochastic model successfully captures the identified $S_{I}$ variation trend, accounting for $94 \%$ of measurements over time within the 0.90 confidence band, and $73 \%$ with a 0.50 confidence. Hence, the model may be slightly conservative, which is safe in control.

When $S_{I}$ increases, the blood glucose level probability interval tightens. The wide range of uncertainties in blood glucose levels associated with low $S_{I}$ reflects the risk of insulin resistant patients under intensive insulin therapy encountering hypoglycaemia [5]. This situation was specifically seen in Patient 4, whose identified $S_{I}$ profile is in the lower physiological range.

\section{Conclusions}

The stochastic model presented defines the distribution of blood glucose levels one hour following a known insulin and/or nutrition intervention. It thus enables more knowledgeable and accurate prediction for glycemic control. The stochastic model acts as a tool to assist clinical intervention decisions, maximising the probability of achieving desired glycemic regulation while maintaining patient safety. The impact of control inputs on probabilistic blood glucose results can be assessed, giving confidence in the effectiveness of the control protocol against evolving patient dynamics.

\section{Reference}

[1] Van den Berghe, G., Wouters, P., Weekers, F., Verwaest, C., Bruyninckx, F., Schetz, M., Vlasselaers, D., Ferdinande, P., Lauwers, P. and Bouillon, R. (2001): 'Intensive insulin therapy in the critically ill patients', $N$ Engl J Med, 345(19), pp. 1359-67.

[2] Van den Berghe, G., Wouters, P. J., Bouillon, R., Weekers, F., Verwaest, C., Schetz, M., Vlasselaers, D., Ferdinande, P. and Lauwers, P. (2003): 'Outcome benefit of intensive insulin therapy in the critically ill: Insulin dose versus glycemic control', Crit Care Med, 31(2), pp. 359-66.

[3] Krinsley, J. S. (2004): 'Decreased mortality of critically ill patients with the use of an intensive glycemic management protocol', SCCM 33rd Annual Congress, Orlando, USA.

[4] Chase, J. G., Shaw, G. M., Lin, J., Doran, C. V., Hann, C., Lotz, T., Wake, G. C. and Broughton, B. (2005): 'Targeted glycemic reduction in critical care using closed-loop control', Diabetes Technol Ther, 7(2), pp. 274-82.

[5] Amiel, S. A., Tamborlane, W. V., Simonson, D. C. and Sherwin, R. S. (1987): 'Defective glucose counterregulation after strict glycemic control of insulin-dependent diabetes mellitus', $N$ Engl J Med, 316(22), pp. 1376-83.

[6] Hann, C. E., Chase, J. G., Lin, J., Lotz, T., Doran, C. V. and Shaw, G. M. (2005): 'Integral-based parameter identification for long-term dynamic verification of a glucose-insulin system model', Comput Methods Programs Biomed, 77(3), pp. 259-70.

[7] Hovorka, R. and Vicini, P. (2001): 'Parameter Estimation', in Carson, E. and Cobelli, C. (Ed): 'Modelling Methodology for Physiology and Medicine', (Academic Press, London), pp. 107-51.

[8] Shaw, G. M., Chase, J. G., Lee, D. S., Bloomfield, M., Doran, C. V. and Lin, J. (2004): 'How high ARE blood glucose levels in intensive care?' ANZICS ASM, Wellington, New Zealand.

[9] Prigeon, R. L., Roder, M. E., Porte, D., Jr. and Kahn, S. E. (1996): 'The effect of insulin dose on the measurement of insulin sensitivity by the minimal model technique. Evidence for saturable insulin transport in humans', J Clin Invest, 97(2), pp. 501-7.

[10] Thorsteinsson, B. (1990): 'Kinetic models for insulin disappearance from plasma in man', Dan Med Bull, 37(2), pp. 143-53.

[11] DeFronzo, R. A., Tobin, J. D. and Andres, R. (1979): 'Glucose clamp technique: a method for quantifying insulin secretion and resistance', Am J Physiol, 237(3), pp. E214-23.

[12] Wong, X. W., Chase, J. G., Shaw, G. M., Hann, C. E., Lin, J. and Lotz, T. (2005): 'Comparison of Adaptive and Sliding-Scale Glycaemic Control in Critical Care and the Impact of Nutritional Inputs', Proc. of the 12th International Conf on Biomedical Engineering (ICBME 2005), Singapore. 